\title{
Assistive technology use and veterans: An examination of racial differences between Whites and Blacks using the HAAT model
}

\author{
Shondra Loggins Clay ${ }^{\mathrm{a}, *}$ and Reginald Alston ${ }^{\mathrm{b}}$ \\ ${ }^{a}$ Research Data Analyst, Counseling Center, University of Illinois at Urbana-Champaign, Champaign, IL, USA \\ ${ }^{\mathrm{b}}$ Department of Kinesiology and Community Health, University of Illinois at Urbana-Champaign, IL, USA
}

Revised/Accepted April 2015

\begin{abstract}
.
BACKGROUND: Many disability researchers have advocated for the use of assistive technology to enhance quality of life for persons with disabilities. However, it has been documented that minorities and veterans are two groups that underutilize the resource.

OBJECTIVE: To use the Human Activity Assistive Technology (HAAT) model to explore assistive technology (AT) use among veterans, specifically examining race, gender, age, socioeconomic determinants (e.g. marital status, educational attainment, employment status, and income), access to health care, general health, and disability status.

METHODS: Data were analyzed from the national 2012 Behavioral Risk Factor Surveillance System (BRFSS). Descriptive statistics, chi-square analyses, and multivariate analyses were performed.

RESULTS: Black veterans used AT more than White veterans, which was consistent with the predictions that indicated that Black veterans were 1.3 times more likely to use AT $(\mathrm{OR}=1.30 \mathrm{CI}$ : $1.20-1.42)$. However, White veterans who used AT had a higher socioeconomic status compared to Black veterans who used AT. More White veterans were married, had higher educational attainment levels, were employed, and had higher income levels. White veterans also had better health coverage, fewer issues with medical costs and better general health. Whereas all of the predictors of AT use were significant for White veterans, only age $(p<0.001)$, employment status $(p<0.001)$, income $(p=0.006)$, medical costs $(p=0.049)$, general health $(p<0.001)$, and limiting disability status $(p<0.001)$ were significant predictors of AT use for Black veterans.

CONCLUSIONS: There are differences in AT use between White and Black veterans based on socioeconomic determinants, access to health care, general health, and disability status. Different predictors and differences in magnitude were observed. Racial differences can partially be explained by components of the HAAT model such as the type of activity that the human is engaging in (e.g. employment) and the context (e.g. the environment).
\end{abstract}

Keywords: Assistive technology, race, veterans, socioeconomic status (SES), disability disparities

\footnotetext{
*Address for correspondence: Shondra Loggins Clay, Ph.D., Research Data Analyst, Counseling Center, University of Illinois at Urbana-Champaign, 610 E. John Street-Office 117, Champaign, IL 61820, USA. Tel.: +1 217300 5371; Fax: +1 217244 9645; E-mail: loggins@illinois.edu.
}

\section{Background}

Since the early 19th century, disabilities have been acknowledged within the United States statutes and regulations with some of the first developments including the establishment of educational institutions for individuals who were deaf and blind (NCLD 2007). However, major legislation for dis- 
ability rights and veterans did not emerge until the 20th century with acts such as the 1918 Smith-Sears Veterans Rehabilitation Act, which established vocational rehabilitation education program during World War I, and the 1943 World War II Disabled Veterans Rehabilitation Act, which provided vocational rehabilitation training for veterans of the war (Reed 1992). Even though such acts were first recognized in the early 20th century, laws for provision of special equipment to assist individuals with disabilities were not acknowledged until the late 20th century. The Technology-Related Assistance for Individuals with Disabilities Act of 1988, which is also known as the Tech Act, was the first major legislative act that recognized assistive technology (AT) as an essential device to increase the quality of life of individuals with disabilities (Public Law 100-407). The act defined AT as: Any item, piece of equipment of system, whether acquired commercially, modified or customized, that is commonly used to increase, maintain or improve functional capabilities of individuals with disabilities (Lewis et al., 2012). The aim of the act was three fold: (1) to increase availability of assistive devices, (2) to ensure greater accessibility through funding opportunities, and (3) to provide educational awareness. Subsequent repeals and reauthorizations of the Tech Act occurred in 1998 and 2004 to expand the original statute.

The Tech Act was partly the result of the startling, yet rising prevalence rates of people with disabilities. In the 2012 Annual Disability Status Report, disability was defined as having one or more of the disabilities from six distinct categories: hearing, visual, cognitive, ambulatory, self-care, or independent living. The prevalence of disability in the United States was 12.1 percent for persons of all ages in 2012. For persons ages 65 to 74, approximately 25 percent had a disability; however, the rate of disability was double (50\%) for persons 75 and older (Erickson et al., 2014).

Even though the overall prevalence rate of disability in the United States was $12 \%$, differences were seen by race. The racial stratification of persons with disabilities indicated that the prevalence of disability for Whites was 10.2 percent; 14.2 percent for Blacks/African Americans; 4.3 percent among Asians; $17.6 \%$ among Native Americans; and 9.9 percent for persons of some other race(s). Other important socioeconomic indicators explored were employment rate, income, educational attainment, and access to health care. The employment rate for persons with disabilities ages 21 to 64 was 33.5 per- cent; the median annual earning for persons with disabilities in the United States was $\$ 36,400$; approximately 34.4 percent of working age persons with disabilities has a high school diploma or equivalent; and the percentage of persons with access to health insurance was 82.8 percent. The percentage of veterans with a Veterans Service-Connected disability was 20.2\% (Erickson et al., 2014).

Many researchers and policy makers have studied the trends in disabilities in the United States and have advocated for the use of assistive devices to enhance the quality of life for persons with disabilities. Furthermore, the benefits of using AT have been duly noted in current research (Agee et al., 2005; Kaye et al., 2008). Despite the voluminous research asserting AT's potentialities and benefits, minorities and veterans are examples of groups that underuse the resource. Strikingly, the same groups that are not fully realizing the benefits of AT have some of the highest prevalence rates of disabilities.

There have been many reasons asserted to potentially explain the disparities in AT use such as a lack of knowledge about the various devices for different types of disabilities, affordability, unawareness of resources that may assist in securing devices, and racial differences in participation of rehabilitation services (Kaye et al., 2008; Lewis, 2009; Loggins, 2014; Alston, 2014). However, it is unclear if the asserted reasons are applicable to the veteran population. Similarly, many scholars have attempted to provide a theoretical basis for exploring AT outcomes in research and practice (Lenker, 2010). However, there has been little systemic effort to incorporate the conceptual models in current research to explain disparities in use of AT, especially in underexplored populations such as minorities and veterans.

One such model that may explain disparities in use of AT is the Human Activity Assistive Technology (HAAT) Model (Cook \& Hussey, 2002). Bailey (1996) first introduced the human performance model; however, Cook revised and expanded the original model to explicitly explore AT. The main components of the HAAT Model include the human being, activity of focus, and AT. The human component is defined as the individual with the disability. Activities reflect human performance (e.g. activities that the human is engaging in), which is based on activities of daily living. AT refers to the device that the individual with the disability uses for human performance (Cook \& Hussey, 2002). The three main components of the HAAT Model interact with the context, another component of the model. Context is 
defined as the environment in which the three main components of the HAAT model interrelate. Components of the context are the social context (e.g. family, colleagues, friends, strangers), the setting (e.g. home, school, employment, community), and the physical context (e.g. space, surroundings, light, sound). The HAAT framework is useful in identifying the main indicators of using AT, but the model is especially exceptional for highlighting the external influence on the utilization of AT by persons with disabilities (Lenker, 2010).

This study will use the HAAT model as a theoretical framework to explore minority veterans and the relationship among use of AT, demographic factors (e.g., age, gender, educational attainment, employment status, and disability status), and socioeconomic determinants (e.g. marital status, income, and access to medical coverage). This study is a follow up to previous investigation that explored the utilization of AT by race using an older 2007 version of the Behavioral Risk Factor Surveillance System (BRFSS). The current study is unique because it utilizes the most recent version of the BRFSS (2012) and it applies a conceptual model for assistive technology to explain AT use in the veteran population. The guiding research questions were: (1) Are there distinct differences in the use of AT for White veterans and Black veterans based on factors such as age, gender, educational attainment, employment status, marital status, income, access to medical coverage, medical costs, general health, and disability status? (2) What are the significant factors that predict the likelihood of using AT for White veterans and Black veterans?

\section{Methods}

\subsection{Setting and study design}

This study retrospectively examined the 2012 Behavioral Risk Factor Surveillance System (BRFSS) dataset. The first BRFSS was established in 1984 and included only 15 states. Today, it has evolved into one of the largest datasets worldwide incorporating data from all 50 states in the United States, the District of Columbia, American Samoa, Guam, Palau, Puerto Rico, and the U.S. Virgin Islands. Through a collaborative effort between the Centers for Disease and Control (CDC) and state health departments, the BRFSS captures behavioral risk factors on an individual level through a survey containing three parts: the main questionnaire, optional modules, and state-added questions. Data are collected via telephone interviews using landlines and cellular phones, which is new feature that was implemented in 2011. More than 500,000 interviews were conducted in 2011 for the most recent version of the BRFSS (2012). Only the main questionnaire was used in this analysis (www.cdc.gov/brfss).

\subsection{Selection of participants}

The original BRFSS assessed several racial groups including Whites, Blacks, Asian, Native Hawaiian or other Pacific Islander, American Indian or Alaskan, Other Race, Multiracial, and Hispanic. Consistent with other research exploring the association of AT use and race (Loggins et al., 2013, Alston et al., 2014), the sampling approach included only targeting White and Black respondents from the general population. Further stratification was performed for most analyses to include only White veterans and Black veterans to test our HAAT model. In the general population, there were a total of 402,761 individuals that were either White or Black, with 52,765 (13.1\%) being veterans. Of the Black and White veterans, 7,912 $(15.0 \%)$ individuals used AT. White veterans that used AT accounted for $90.4 \%(n=7,154)$ and $9.6 \%$ $(n=758)$ were Black veterans that used AT.

\subsection{Factors}

\subsubsection{Veteran status}

Veteran status in the main questionnaire of the BRFSS 2012 was assessed by asking participants the following question: "Have you ever served on active duty in the United States, Armed Forces, either in the regular military or in a National Guard or military reserve unit? Active duty does not include training for the Reserves or National Guard, but does include activation, for example, for the Persian Gulf War." Veteran status was measured as a dichotomous variable $(1=$ yes and $0=$ no $)$. All variables coded as don't know or not sure were excluded from this analysis. In this study, veteran status was also operationalized as the "human" (or the person using the assistive device or doing the activity) from the HAAT model.

\subsubsection{Assistive technology use}

In the original and new dataset, AT was measured by the following survey question: "Do you now have any health problem that requires you to use special equipment, such as a cane, a wheelchair, a special bed, or a special telephone? (Include occasional use or use 
in certain circumstances)." AT use was measured as a categorical variable formatted as: $1=$ yes and $0=$ no. If a participant indicated "yes," as a response to the question, he/she was considered to use AT. In this study and the HAAT model, AT was defined as the device that the individual with the disability uses to perform the activity.

\subsubsection{Gender}

The original question from the 2012 BRFSS main survey assessed gender through the statement: "indicate the sex of the respondent." In the original and the new dataset, gender was measured as a dichotomous variable with the possible responses of $1=$ males and $0=$ females. Gender represented another "human component" as defined in the HAAT model.

\subsubsection{Marital status}

Marital status was formatted by 6 possible answer choices in the original dataset: married, divorced, widowed, separated, never married, or a member of an unmarried couple. In the original and the new dataset, a participant was identified as married if he/she responded to the answer choice "married." In the new dataset, the category, "not married," was created and was defined as either divorced, widowed, separated, never married, or member of an unmarried couple to create a dichotomous variable $(1=$ married and $0=$ not married). Marital status was explored as the "context" from the HAAT model.

\subsubsection{Educational attainment}

In the original main questionnaire of the 2012 BRFSS, educational attainment was assessed using categories of never attended school or only kindergarten, grades 1 through 8 (elementary), grades 9 through 11 (some high school), grade 12 or GED (high school graduate), college 1 year to 3 years (some college or technical school), and college 4 years or more (college graduate). The education variable was recoded to reflect $1=$ HS education or higher and $0=$ less than a HS education. High education levels was defined as being a high school graduate, having some college or technical school, or being a college graduate. Educational attainment was examined as an example of "context" from the HAAT model.

\subsubsection{Employment status}

The employment variable was originally defined in the BRFSS by several nominal depictions. Respondents were allowed to choose from categories such as employed for wages, self-employed, out of work for more than 1 year, or out of work for less than 1 year. Other options for employment status included being a homemaker or being a student, and being retired or unable to work. In this study, employment was recoded to indicate $1=$ employed and $0=$ not employed. A participant was identified as having "employed" status if she/he selected one of the following categories: employed for wages, self-employed, or self identified their status as a homemaker. Employment status was explored as an instance of "activity" from the HAAT model.

\subsubsection{Income level}

The BRFSS main questionnaire measured the income variable by using categories of less than $\$ 10,000$, less than $\$ 15,000(\$ 10,000-\$ 15,000)$, less than $\$ 20,000(\$ 15,000-\$ 20,000)$, less than $\$ 25,000$ $(\$ 20,000-\$ 25,000)$, less than $\$ 35,000(\$ 25,000$ $\$ 35,000)$, less than $\$ 50,000(\$ 35,000-\$ 50,000)$, less than $\$ 75,000(\$ 50,000-\$ 75,000)$, and more than $\$ 75,000$. In this study, income was formatted based on the U.S. 2014 poverty guidelines as: $1=$ more than $\$ 25,000$ and $0=$ less than $\$ 25,000$. The 2014 poverty guidelines for the 48 contiguous states and the District of Columbia were updated annually based on the individuals in the household. For one person listed in the family/household, the current poverty level is $\$ 11,670$. The dollar amount for household income increases by $\$ 4,060$ for each additional person. For a family/household of 4 individuals, the poverty level was $\$ 23,850$, which was used as the cutoff income level for this study (www.aspe.hhs.gov). Income was defined as "context" from the HAAT model.

\subsubsection{Health coverage}

Health Coverage was formatted as a categorical variable in the old and new dataset containing two options: $1=$ have health coverage and $0=$ do not have health coverage. A participant was identified as having health coverage if the respondent selected "yes" as the answer choice to the following question: "Do you have any kind of health care coverage, including health insurance, prepaid plans such as HMOs or government plans such as Medicare?" Health coverage was operationalized as "context" from the HAAT model.

\subsubsection{Medical cost}

Participants were asked: "Was there a time in the past 12 months when you needed to see a doctor but could not because of cost?" In this study, medical 
cost was formatted as $1=$ medical costs not an issue and $0=$ medical cost an issue and assessed individualized affordability. Unaffordable health coverage was determined if the participant chose the response "yes" in the original dataset to the previously mentioned question. In the new dataset, if an individual answered "yes", it was recoded as medical cost is an issue; a response of "no" indicated that medical cost was not an issue. Medical cost was used as a "context" from the HAAT model.

\subsubsection{General health}

General health was originally measured using categories of excellent, very good, good, fair, and poor. General health was recoded as a dichotomous variable $(1=$ general health good or better and $0=$ general health fair or worse). A participant was considered to have good health if he/she had good, very good, or excellent health. General health was an example of the "human component" in the HAAT model.

\subsubsection{Limiting disability status}

Disability status was measured as a dichotomous variable in the original and new data set. The question in the BRFSS asked: Are you limited in any way in any activities because of physical, mental, or emotional problems? The possible answers were yes or no. The codes in the new dataset for disability status were: $1=$ have a disability that limits activity and $0=$ do not have a disability that limits activity. Limiting disability status was defined as a "human" factor in the HAAT model as well.

\subsubsection{Age}

Age was identified as a continuous variable in the original 2012 main questionnaire of the BRFSS and the new dataset. The survey question asked was: "What is your age?" In the original dataset, the answers ranged from the age of 7 to 99 years. A targeted sampling approach was adapted to include only adults ranging from the age of 18 years to 99 years. The mean age of the general population was $56.32(\mathrm{SD}=17.3)$, whereas the mean age of veterans was $64.51(\mathrm{SD}=15.4)$. Veterans who used AT had a mean age of $70.72(\mathrm{SD}=13.5)$. In this study, age was operationalized as a part of the "human component" in the HAAT model.

\subsection{Statistical analysis}

Data analyses were performed using the most current version of the IBM SPSS 22.0 statistical software program. Descriptive statistics were performed to describe the sample including the general population and the veteran population. Differences between Black and White veterans across race, gender, age, socioeconomic determinants (e.g. marital status, educational attainment, employment status, income levels), health coverage, medical cost, general health, and disability status were assessed using Chisquare analyses. Four individual models of logistic regressions were conducted to estimate the probability of using AT. Logistic regression models to predict the use of AT were conducted for the general population $(n=402,761)$, veterans $(n=52,765)$, White veterans $(n=7,154)$, and Black veterans $(n=758)$. Finally, multivariate nested models were used to explore and control for the exposure to resources from the HAAT model to determine if the variables assessed account for the difference in AT use between Black and White veterans.

\section{Results}

Results from the descriptive statistics, the chisquare statistical analyses, and the logistic regression models are discussed in this section. Specifically, the guiding research questions exploring differences in the use of AT for veterans based on race and predictive factors are addressed.

\subsection{Descriptive statistics}

The BRFSS 2012 dataset contained almost 403,000 individual cases in the general population that were either Black or White. There were 363,139 Whites $(90.2 \%)$ and 39,622 (9.8\%) Blacks. Of individuals in the general population that were either White or Black, most were female $(60.1 \%$,), married $(53.1 \%)$, had a HS education or higher $(92.6 \%)$, employed (55.5\%), had an income of more than $\$ 25,000(62.0 \%)$, had health coverage (89.8\%), claimed that medical cost was not an issue $(88.6 \%)$, had a general health status of good or better $(81.2 \%)$, and did not have a disability that limited activity (73.4\%). Additionally, about 46,015 (11.4\%) individuals used AT and the mean age of the general population was $56.32(\mathrm{SD}=17.263)$.

The descriptive statistics from the population of interest (veterans) were similar to the general population. There were 52,765 veterans that were either White $(92.1 \%, n=48,605)$ or Black $(7.9 \%$. $n=4,160)$. Similar to the general population, most 
veterans were married (61.2\%), had a HS education or higher (94.8\%), had income levels above $\$ 25,000$ (68.6\%), had health coverage $(93.8 \%)$, stated that medical cost was not an issue $(93.3 \%)$, had a general health status of good or better (77.8\%), and did not have a disability that limited activity (68.7\%). However, in contrast to the general population, most veterans were male $(91.8 \%)$, and were not employed $(61.9 \%)$. A total of $15.0 \%(n=7,912)$ of the veteran population used AT. The mean age of the veteran population was $64.51(\mathrm{SD}=15.399)$.

Even though there could be substantive differences between individuals who have veteran status and individual who have veteran status and use AT, in our sampled population, the demographic characteristics of the two groups were similar. Of the veteran population who used AT $(15 \%, n=7,912)$, most were White $(90.4 \%)$. Irrespective of race, most were also male (92.2\%), married (51.2\%), had a high school education or higher $(91.6 \%)$, were not employed (87.9\%), had income more than $\$ 25,000$ (51.4\%), had health coverage (94.8\%), and stated that medical costs were not an issue (89.4\%). In contrast to veterans in general, most veterans who used AT claimed that their general health was fair or worse $(54.8 \%)$, and had a disability that limited activity $(74.8 \%)$ (see Table 1 ).

\subsection{Racial differences in the use of assistive technology for veterans}

Racial differences for White veterans and Black veterans that used AT were revealed based on gender, age, socioeconomic determinants (e.g. marital status, educational attainment, employment status, income), access to health coverage, medical costs, and general health. Disability status was the only variable that did not yield a significance difference by race.

After examining the proportion of AT use for veterans by race, $14.9 \%$ of White veterans used AT compared to $18.6 \%$ of Black veterans $(p<0.001)$, indicating a higher use of AT by Blacks compared to Whites. Most variables such as gender, educational attainment, employment status, access to health coverage, medical costs, and general health were the same across group dynamic categories and race but differed in proportions. White and Black veterans who were males used AT more than females; however, the proportion of White male veterans who used AT was $92.8 \%$ compared to $86.9 \%$ for Black male veterans $(p<0.001)$. In general, individuals who had a HS education or higher used AT more than those with less than a HS education. However, a slightly higher percentage of White veterans (92\%) who used AT had at least a high school education in comparison to $87.3 \%$ of Black veterans $(p<0.001)$. Veterans who were not employed used AT more than those who were employed; however, within proportion differences revealed that $87.8 \%$ of White veterans who use AT were unemployed compared to $90.6 \%$ of Black veterans $(p=0.024)$. Differences were also observed in access to health coverage and medical costs. For Whites veterans who used AT, 95.6\% had health coverage compared to $89.2 \%$ of Black veterans. Most veterans who used AT stated that medical costs were not an issue; however, for White veterans who used AT, $90.5 \%$ claimed that medical costs were not an issue compared to $82.5 \%$ of Black veterans $(p<0.001)$. Similarly for White veterans who used AT, $54.7 \%$ stated that their general health was fair or worse compared to $60.1 \%$ of Black veterans who used AT $(p=0.005)$.

In contrast, the proportion of White female veterans who used AT was $7.2 \%$ compared to $13.1 \%$ for Black female veterans; White veterans who used AT with less than a HS education accounted for $7.8 \%$ compared to Black veterans who used AT with less than a HS education which was $12.7 \%$; White veterans who use AT who were employed was $12.2 \%$ compared to $9.4 \%$ of Black veterans; Whites veterans who used AT who did not have health coverage was $4.4 \%$ compared to $10.8 \%$ of Black veterans; White veterans who used AT and claimed that medical costs were an issue was $9.5 \%$ compared to $17.5 \%$ of Black veterans; and White veterans who used AT who stated that their general health was good or better was $45.3 \%$ compared to $39.9 \%$ of Black veterans who used AT.

Other variables that showed statistical significant racial differences were marital status $(p<0.001)$, income $(p<0.001)$, and age $(p<0.001)$. In contrast to the other variables that were statistically significant, group dynamics differed. Most White veterans who used AT were married (52.8\%) while most Black veterans who used were not married (62.9\%). Similarly, most White veterans who used AT made more than $\$ 25,000$ (59.7\%); however, most Black veterans who used AT made less than $\$ 25,000$ (55.9\%). The average age of White veterans who used AT was $71.45(\mathrm{SD}=13.4)$, whereas the average age of Black veterans who used AT was $63.86(\mathrm{SD}=13.1)$ (see Table 1)..

Overall, the exploration of the relationship of AT use and veterans revealed prominent racial differences between White and Black veterans based on socioeconomic determinants, access to health care, 
perception of medical costs, and general health. White veterans who used AT had a higher socioeconomic status compared to Black veterans who used AT. More White veterans were married, had higher educational attainment levels, were employed, and had higher income levels. White veterans also had better health coverage, fewer issues with medical costs and better general health.

\subsection{Predictive factors in the use of AT for veterans}

Four binary logistic regression analyses were performed to explore potential factors associated with AT use. The independent factors examined in the logistic regression analyses were: veteran status, race, gender, marital status, educational attainment, employment status, income levels, health coverage, medical costs, general health, disability status, and age. The dependent variable was AT use. In the first model, potential predictors of AT use for the general population (e.g. Whites and Blacks) were explored (Table 2); the second model assessed predictors for veterans (only Whites and Blacks) (Table 2); the third model examined potential predictors for White veterans only (Table 3); and the fourth model explored predictors for Black veterans only (Table 3 ).

In the general population (e.g. all Whites and Blacks), significant factors that had a positive association to AT use were being a veteran $(\mathrm{OR}=1.04$, CI: $1.00-1.09, p=0.046)$, being Black $(\mathrm{OR}=1.57$, CI: $1.51-1.64, p<0.001)$, having health coverage $(\mathrm{OR}=1.58$, CI: $1.50-1.67, p<0.001)$, having a limiting disability $(\mathrm{OR}=8.20, \mathrm{CI}: 7.97-8.45, p<0.001)$, and older age $(\mathrm{OR}=1.04$, CI: $1.04-1.04, p<0.001)$. Other significant factors that had a negative association to AT use were being male $(\mathrm{OR}=0.96, \mathrm{CI}$ : $0.93-0.99, p=0.012)$, being married $(\mathrm{OR}=0.82, \mathrm{CI}$ : $0.80-0.85, p<0.001)$, being employed $(\mathrm{OR}=0.53$, CI: $0.51-0.55, p<0.001)$, having income more than $\$ 25,000$ (OR $=0.66$, CI: 0.64-0.68, $p<0.001$ ), medical costs not an issue $(\mathrm{OR}=0.88$, CI: $0.85-0.92$, $p<0.001)$, and general health good or better (OR $=0.38$, CI: $0.36-0.39, p<0.001)$.

In the second logistic regression model which explored AT use in the veteran population (only White and Black veterans), similar results to the general population were observed. The same variables that showed a significant positive association to AT use in the general population (e.g. being Black, having health coverage, having a limiting disability, and older age) showed a positive association to AT use in the veterans population; likewise, the variables that showed a significant negative association to AT use in the general population (e.g. being male, being married, being employed, having an income more than $\$ 25,000$, medical costs not an issue, and general health good or better) showed a negative association to AT use in the veterans population. The only difference between the general population and veterans was the estimates of probability. Significant estimates that differed by a magnitude of more than $5 \%$ between the general population and veterans were being male $(\mathrm{OR}=0.83$, CI: $0.74-0.94, p=0.002)$, being married $(\mathrm{OR}=0.89$, CI: $0.84-0.95, p<0.001)$, having health coverage $(\mathrm{OR}=1.25$, CI: $1.09-1.44$, $p=0.002)$, medical costs not an issue $(\mathrm{OR}=0.78, \mathrm{CI}$ : $0.70-0.87, p<0.001)$, and having a limiting disability $(\mathrm{OR}=6.59$, CI: 6.17-7.04, $p<0.001$ ) (see Table 2).

After examining the predictors of AT use by race, differences were observed for White veterans and Black veterans. Similar to the general population and the veteran population, significant factors that had a positive association to AT use for White veterans were having health coverage $(\mathrm{OR}=1.28$, CI: $1.09-1.49$, $p=0.002)$, having a limiting disability $(\mathrm{OR}=6.47$, CI: $6.04-6.93, p<0.001)$, and older age (OR $=1.03$, CI: $1.02-1.03, p<0.001)$. Other significant factors that had a negative association to AT use were being male $(\mathrm{OR}=0.81, \mathrm{CI}: 0.71-0.92, p=0.001)$, being married $(\mathrm{OR}=0.90, \mathrm{CI}: 0.84-0.96, p=0.002)$, being employed (OR $=0.52$, CI: $0.47-0.57, p<0.001)$, having income more than $\$ 25,000(\mathrm{OR}=0.69, \mathrm{CI}$ : $0.65-0.75, p<0.001)$, medical costs not an issue $(\mathrm{OR}=0.78$, CI: $0.70-0.88, p<0.001)$, and general health good or better $(\mathrm{OR}=0.40, \mathrm{CI}$ : $0.37-0.42$, $p<0.001)$. The difference between the general population and White veterans was with educational attainment. For White veterans, having a HS education or higher was positively associated with AT use (OR $=1.19$, CI: $1.04-1.35, p=0.009)$.

Black veterans had fewer predictors that were associated with AT use compared to White veterans. Even though the direction of the association was the same for Black and White veterans, the magnitude of the estimates differed. The only variables that were positively associated with AT use for Black veterans were having a limiting disability $(\mathrm{OR}=7.70, \mathrm{CI}: 6.16-9.62, p<0.001)$, and older age $(\mathrm{OR}=1.02$, CI: $1.01-1.03, p<0.001)$. Variables that were negatively associated to AT use were being employed (OR $=0.29$, CI: $0.21-0.40, p<0.001)$, having income more than $\$ 25,000(\mathrm{OR}=0.72, \mathrm{CI}$ : $0.57-0.91, p=0.006)$, medical costs not an issue 


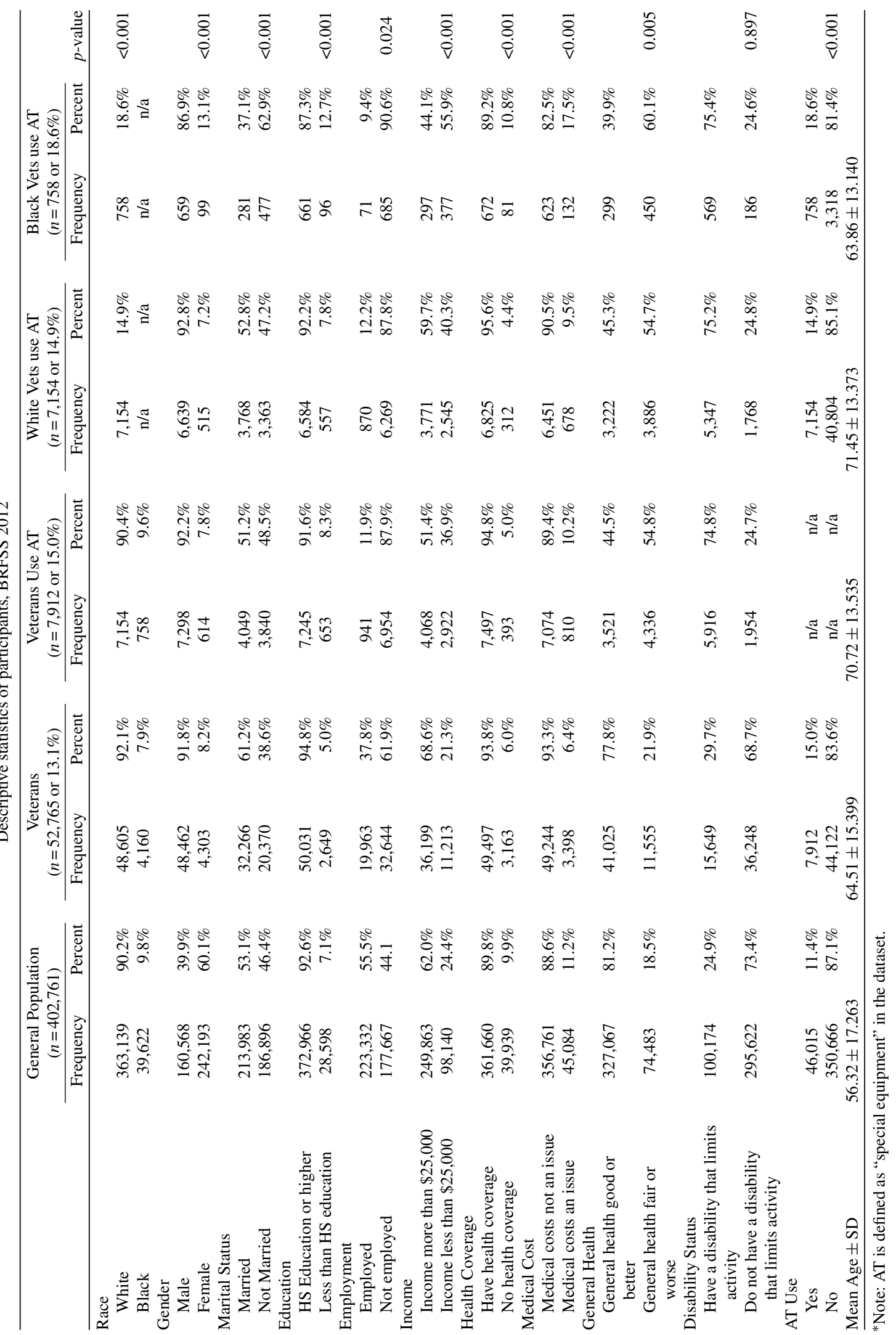


Table 2

Predictors of AT use: General population versus Veterans. BRFSS 2012

\begin{tabular}{|c|c|c|c|c|c|c|c|c|}
\hline \multirow[b]{4}{*}{ Veteran $($ ref $=$ not a Veteran $)$} & \multicolumn{8}{|c|}{ Use AT } \\
\hline & \multicolumn{4}{|c|}{ General Population } & \multicolumn{4}{|c|}{ Veterans } \\
\hline & \multirow{2}{*}{$\begin{array}{c}\text { OR } \\
1.04\end{array}$} & \multicolumn{2}{|c|}{$95 \% \mathrm{CI}$} & \multirow{2}{*}{$\frac{p \text {-value }}{0.046}$} & \multirow[t]{2}{*}{$\overline{\mathrm{OR}}$} & \multicolumn{2}{|c|}{$95 \% \mathrm{CI}$} & \multirow[t]{2}{*}{$\overline{p \text {-value }}$} \\
\hline & & 1.00 & 1.09 & & & & & \\
\hline Black $($ ref $=$ White $)$ & 1.57 & 1.51 & 1.64 & $<0.001$ & 1.55 & 1.40 & 1.73 & $<0.001$ \\
\hline Males $($ ref $=$ female $)$ & 0.96 & 0.93 & 0.99 & 0.012 & 0.83 & 0.74 & 0.94 & 0.002 \\
\hline Married (ref $=$ not married) & 0.82 & 0.80 & 0.85 & $<0.001$ & 0.89 & 0.84 & 0.95 & 0.001 \\
\hline HS education or higher (ref $=$ less than HS education) & 0.98 & 0.94 & 1.02 & 0.239 & 1.12 & 1.00 & 1.27 & 0.054 \\
\hline Employed $(\mathrm{ref}=$ not employed $)$ & 0.53 & 0.51 & 0.55 & $<0.001$ & 0.49 & 0.45 & 0.54 & $<0.001$ \\
\hline Income $>\$ 25,000(\mathrm{ref}=$ income $<\$ 25,000$ & 0.66 & 0.64 & 0.68 & $<0.001$ & 0.70 & 0.65 & 0.75 & $<0.001$ \\
\hline Health coverage $(\mathrm{ref}=$ no health coverage $)$ & 1.58 & 1.50 & 1.67 & $<0.001$ & 1.25 & 1.09 & 1.44 & 0.002 \\
\hline Medical costs not an issue (ref $=$ medical costs an issue $)$ & 0.88 & 0.85 & 0.92 & $<0.001$ & 0.78 & 0.70 & 0.87 & $<0.001$ \\
\hline General health good or better (ref = general health fair or worse) & 0.38 & 0.36 & 0.39 & $<0.001$ & 0.40 & 0.38 & 0.43 & $<0.001$ \\
\hline Limiting Disability (ref $=$ do not have a limiting disability) & 8.20 & 7.97 & 8.45 & $<0.001$ & 6.59 & 6.17 & 7.04 & $<0.001$ \\
\hline Age & 1.04 & 1.04 & 1.04 & $<0.001$ & 1.03 & 1.02 & 1.03 & $<0.001$ \\
\hline
\end{tabular}

Table 3

Predictors of AT use by race: White veterans versus Black veterans. BRFSS 2012

\begin{tabular}{|c|c|c|c|c|c|c|c|c|}
\hline \multirow[b]{4}{*}{ Males (ref $=$ female $)$} & \multicolumn{8}{|c|}{ Use AT } \\
\hline & \multicolumn{4}{|c|}{ White Veterans } & \multicolumn{4}{|c|}{ Black Veterans } \\
\hline & \multirow{2}{*}{$\begin{array}{c}\text { OR } \\
0.81\end{array}$} & \multicolumn{2}{|c|}{$95 \% \mathrm{CI}$} & \multirow{2}{*}{$\frac{p \text {-value }}{0.001}$} & \multirow{2}{*}{$\begin{array}{c}\text { OR } \\
0.92\end{array}$} & \multicolumn{2}{|c|}{$95 \% \mathrm{CI}$} & \multirow{2}{*}{$\begin{array}{r}p \text {-value } \\
0.619\end{array}$} \\
\hline & & 0.71 & 0.92 & & & 0.67 & 1.27 & \\
\hline Married $($ ref $=$ not married $)$ & 0.90 & 0.84 & 0.96 & 0.002 & 0.83 & 0.67 & 1.04 & 0.098 \\
\hline HS education or higher ( $\mathrm{ref}=$ less than HS education) & 1.19 & 1.04 & 1.35 & 0.009 & 0.74 & 0.52 & 1.06 & 0.099 \\
\hline Employed $($ ref $=$ not employed $)$ & 0.52 & 0.47 & 0.57 & $<0.001$ & 0.29 & 0.21 & 0.40 & $<0.001$ \\
\hline Income $>\$ 25,000(\mathrm{ref}=$ income $<\$ 25,000$ & 0.69 & 0.65 & 0.75 & $<0.001$ & 0.72 & 0.57 & 0.91 & 0.006 \\
\hline Health coverage $(\mathrm{ref}=$ no health coverage $)$ & 1.28 & 1.09 & 1.49 & 0.002 & 1.17 & 0.83 & 1.65 & 0.368 \\
\hline Medical costs not an issue (ref $=$ medical costs an issue $)$ & 0.78 & 0.70 & 0.88 & $<0.001$ & 0.73 & 0.54 & 1.00 & 0.049 \\
\hline General health good or better ( $\mathrm{ref}=$ general health fair or worse) & 0.40 & 0.37 & 0.42 & $<0.001$ & 0.48 & 0.39 & 0.60 & $<0.001$ \\
\hline Limiting Disability (ref $=$ do not have a limiting disability) & 6.47 & 6.04 & 6.93 & $<0.001$ & 7.70 & 6.16 & 9.62 & $<0.001$ \\
\hline Age & 1.03 & 1.02 & 1.03 & $<0.001$ & 1.02 & 1.01 & 1.03 & $<0.001$ \\
\hline
\end{tabular}

Table 4

Multivariate nested model for predictors of AT use for veterans based on the HAAT Model. BRFSS (2012)

\begin{tabular}{|c|c|c|c|c|c|}
\hline Variables & $\begin{array}{l}\text { Model } 1 \\
\text { Baseline }\end{array}$ & $\begin{array}{c}\text { Model } 2 \\
\text { human }\end{array}$ & $\begin{array}{l}\text { Model } 3 \\
\text { activity }\end{array}$ & $\begin{array}{c}\text { Model } 4 \\
\text { context }\end{array}$ & $\begin{array}{c}\text { Model } 5 \\
\text { full }\end{array}$ \\
\hline \multicolumn{6}{|l|}{ Race } \\
\hline Black (ref = White) & $1.30^{* *}$ & $1.70^{* *}$ & $1.62^{* *}$ & $1.61^{* *}$ & $1.55^{* *}$ \\
\hline \multicolumn{6}{|l|}{ Human Component } \\
\hline Males $($ ref $=$ female $)$ & & $0.78^{* *}$ & $0.74^{* *}$ & $0.88^{*}$ & $0.83^{* *}$ \\
\hline General health good or better ( $\mathrm{ref}=$ general health fair or worse) & & $0.34^{* *}$ & $0.37^{* *}$ & $0.38^{* *}$ & $0.40^{* *}$ \\
\hline Limiting Disability (ref $=$ do not have a limiting disability) & & $7.28^{* *}$ & $6.75^{* *}$ & $7.06^{* *}$ & $6.59^{* *}$ \\
\hline Age & & $1.04^{* *}$ & $1.04^{* *}$ & $1.04^{* *}$ & $1.03^{* *}$ \\
\hline \multicolumn{6}{|l|}{ Activity } \\
\hline Employed (ref = not employed) & & & $0.45^{* *}$ & & $0.49^{* *}$ \\
\hline \multicolumn{6}{|l|}{ Context } \\
\hline Married (ref = not married) & & & & $0.88^{* *}$ & $0.89^{* *}$ \\
\hline HS education or higher (ref $=$ less than HS education) & & & & $1.14^{*}$ & $1.12^{\ddagger}$ \\
\hline Income $>\$ 25,000($ ref $=$ income $<\$ 25,000$ & & & & $0.63^{* *}$ & $0.70^{* *}$ \\
\hline Health coverage (ref $=$ no health coverage $)$ & & & & $1.27^{* *}$ & $1.25^{* *}$ \\
\hline Medical costs not an issue ( $\mathrm{ref}=$ medical costs an issue $)$ & & & & $0.78^{* *}$ & $0.78^{* *}$ \\
\hline
\end{tabular}

$p<0.10^{\ddagger} ; p<0.05^{*} ; p<0.01^{* *}$. Source: BRFSS 2012.

(OR=0.73, CI: $0.54-1.00, p=0.049)$, and general health good or better (OR $=0.48, \mathrm{CI}$ : $0.39-0.60$, $p<0.001)$. The most substantial difference in magnitude was observed in employment status and having a limiting disability. White veterans who were employed were $48 \%$ less likely to use AT than those who were not employed, whereas Black veterans who were employed were $71 \%$ less likely to use AT than 
those who were not employed. Similarly, White veterans who had a limiting disability were almost 6.5 times more likely to use AT compared to those who do not have a limiting disability; however, Black veterans who had a limiting disability were 7.7 times more likely to use AT compared to those who do not have a limiting disability (see Table 3 ).

\subsection{Multivariate nested model for AT use}

Multivariate nested models were employed to assess differences between Black veterans and White veterans in the use of AT as we controlled for the various components from the HAAT model (e.g. activity and the context). In each model, we explored how the odds ratio for race changed with the addition of variables from the HAAT model. In the bivariate baseline model (Model 1), differentials in the use of AT for Black veterans were compared to White veterans. Model 2 adds the variables that are operationalized as the "human" component from the HAAT model without controlling for any other factors (e.g. activity and the context). This model includes gender, general health status, and limiting disability. Model 3 adds employment status, which is defined as "activity" in the HAAT model. Model 4 includes the "context" variables from the HAAT model such as marital status, educational attainment, income, health coverage, and medical costs (see Table 4).

In the baseline model, Black veterans were 1.3 times more likely to use AT compared to White veterans. In model 2, when the "human" characteristics are added from the HAAT model, the odds ratio (OR) increases to 1.70 . In models 3 , we control for the "activity" component of the HAAT model which is measured by employment. The OR for race decreases (moving towards 1) as we control for components from the HAAT model. Black veterans have 1.62 times the odds of using AT compared to White veterans when "activity" is taken into account. Similarly, in model 4, Black veterans have 1.61 times the odds of using AT when taking the "context" variables into account.

\section{Discussion}

This study was conducted as a follow up to a previous study which explored the utilization of AT by race (Loggins et al., 2014). Similar variables were explored in the current study with the addition of new variables such as marital status, general health, and disability status. Additionally, the unit of analysis differed with the current study specifically exploring the use of AT in the veteran population.

The results from this study revealed that there are distinct differences in the use of AT for White veterans and Black veterans based on factors such as age, gender, educational attainment, employment status, marital status, income, access to medical coverage, medical costs, and disability status. Black veterans used AT more than White veterans which was consistent with the predictions from the logistic regression analyses that indicated Black veterans were 1.3 times more likely to use AT compared to White veterans $(\mathrm{OR}=1.30 \mathrm{CI}: 1.20-1.42)$. The finding of Blacks using AT more than Whites was consistent with previous studies (Loggins et al., 2013); however, it contrasts some studies reporting higher use of assistive devices for Whites (Tomita et al., 1997).

Differences in the utilization of AT among veterans may be partially explained by the three main tenets of the HAAT model: the human, the activity, and the device, which interacts interchangeably. In the HAAT model, the human component examines the person, which in this study we explored veterans; the activity assesses what the human is doing (e.g. what the veteran is doing); and the device explores what specific AT is needed to do the activity. When exploring the veteran population, it may be necessary to explore the specific needs of veterans (e.g. the "human" from the HAAT model) including the type of disability. Murdoch et al. (2003) found that Black veterans had a substantially lower rate of service connected PTSD compared to other veterans. Whereas White veterans may be more impacted by mental disorders, Black veterans may have higher rates of other types of disabilities that may require the use of AT (e.g. physical impairment, auditory impairments, and visual impairments).

It is also important to consider the type of assistive device that is being assessed when exploring racial differences. Resnik And Allen (2006) found that Blacks were 1.2 times more likely than other racial groups to use devices for mobility. In a subsequent study, Corman (2008) also assessed the use of mobility devices for physical impairments (e.g. canes, wheelchairs, and walkers) and found similar results supporting a greater use amongst Blacks compared to Whites. It is possible that White veterans may be more impacted my mental disorders such as PTSD opposed to impairments that require a greater need of assistive devices such as physical impairments for 
mobility. If more veterans in this dataset had physical impairments, it may explain the greater use of AT of Black veterans compared to White veterans.

White and Black veterans differed on many variables in this study. Specifically, more White veterans were married, had higher educational attainment levels, were employed, and had higher income levels. White veterans also had better health coverage, fewer issues with medical costs and better general health. The racial disparities observed between White and Black veterans may be explained from the social context construct of the HAAT model. The social context construct explores external factors that may influence the use of AT. From the findings we can infer that whereas White veterans have strong social factors that may assist in using AT, Black veterans may need external support. Alston et al. (2014) found that a higher percentage of Black veterans compared to White veterans within population received governmental support from Medicaid (19.1\% for Blacks compared to $15.1 \%$ for Whites). Similarly, a higher percentage of Black veterans received SSI $(11.8 \%$ of Blacks compared to $8.5 \%$ of Whites), SSDI (27.3\% for Black veterans compared to $26.7 \%$ for White veterans), and Veteran Benefits (10.2\% for Blacks versus $8.8 \%$ for Whites) (Alston, 2014). The greater access to governmental resources makes it less challenging for Black veterans to obtain assistive devices, especially those who may not be married, have less education, may not be employed, make less money, have worse health coverage, and have more issues with medical costs.

Results from the multivariate nested model also supported this claim. In the baseline model, Black veterans were 1.3 times more likely to use AT compared to White veterans. After adding "human characteristics" (e.g. gender, general health, limiting disability, and age) from the HAAT model, the OR for Black veterans increased to 1.70. The results suggest that when differences in the "human" characteristics of Black and White veterans are taken into consideration, Blacks are still 1.7 times more likely to use AT compared to White veterans. However, in the subsequent models, we controlled for "activity" (e.g. employment) and "context" (e.g. marital status, educational attainment, income, health coverage, and medical costs) from the HAAT model. The ORs increasingly become smaller when controlling for "activity" and "context" after the second model 1. The results indicate that the differences in "activity" and "context" partially explain the racial gap between Black and White veterans. In Model 4, we found a positive association of AT use to having a HS education or higher and having access to health coverage, with both variables increasing the odds of using AT. This is not surprising. However, as mentioned previously, White veterans had a greater proportion of individuals who were more educated and had greater access to health coverage. The results infer that if equity exists across educational attainment levels and accessibility to health coverage (e.g. Black veterans had the same proportions as White veterans for education and health coverage), we may observe an even larger racial gap and more utilization of AT among Black veterans.

The racial differences between White veterans and Black veterans were even more prevalent and consistent in the disaggregated predictive factors findings. Whereas all of the predictors of AT use were significant for White veterans, only age, employment status, income, medical costs, general health, and disability status were significant predictors of AT use for Black veterans. The predictive factors that applied to White veterans but not Black veterans were gender, marital status, educational attainment, and health coverage. For the predictive factors that were similar for White veterans and Black veterans, magnitudes of differences were observed. Employed White veterans were $48 \%$ less likely to use AT compared to those who were not employed; however, employed Black veterans were $71 \%$ less likely to use AT compared to those who were not employed. For Whites, there was a smaller gap between the association of veterans who were employed versus those who were not employed and the utilization of AT compared to Black veterans where there was a much larger gap. The disparity may be explained by three components of the HAAT model: the activity, the AT device, and the social context. Depending on the type of employment (social context) that the veteran has, the individual may be unaware of what type of AT device can be used to assist with the activity or their work related responsibilities. The lack of knowledge about ATs and the related benefits (Alston, 2014; Kaye et al., 2008; Lewis, 2009; Loggins, 2014) may explain this disparity gap in utilization. Similarly, employed veterans may not receive as many of the governmental benefits as unemployed veterans such as SSI, SSDI, or veteran benefits. The lack of exposure to resources may lead to unawareness of where to obtain devices.

Magnitude differences between predictive factors for White veterans and Black veterans were also observed in the relationship of disability status and the use of AT. White veterans who had a limiting dis- 
ability were almost 6.5 times more likely to use AT compared to those who do not have a limiting disability; however, Black veterans who had a limiting disability were 7.7 times more likely to use AT compared to those who do not have a limiting disability. There were two implications from the findings. In contrast to typical outcomes, Black veterans with disabilities have higher estimates of using AT compared to White veterans. The results are intriguing, suggesting that veterans, irrespective of race and who have a documented disability that limits activity, are taking advantage of assistive devices that can improve quality of life and assist with activities of daily living. Rehabilitation leaders, policy makers, and disability advocates should continually encourage use of AT for persons with disabilities, particularly among Black veterans with disabilities.

Similar to most studies that implore secondary data analyses techniques and use predefined datasets to retrospectively explore variables of interest, in this study, we were very limited to the data (e.g. sample size) and the specific questions that were already asked in the 2012 version of the BRFSS. Additionally, data specific to veterans were very limited. Overall, there were a very limited amount of quantitative questions asked that limited our analysis. The only quantitative variable used that was applicable to our interest was "age." We would have liked to analyze other quantitative variables that could possibly be predictors of AT use such as time served in the military and amount of medical coverage. The addition of other variables that we hypothesize that will have an association to AT use will expand this study and create a true empirical examination of AT use for veterans. Therefore, future research should attempt to incorporate data with more quantitative data and data specific to the veteran population.

Another limitation of the dataset was the extent of the questions asked. Two questions in this analysis that were ambiguous were health equipment and limiting disability. The variable health equipment was operationalized through the question: "Do you now have any health problem that requires you to use special equipment, such as a cane, a wheelchair, a special bed, or a special telephone? (Include occasional use or use in certain circumstances)." Similarly, limiting disability was defined as: "Are you limited in any way in any activities because of physical, mental, or emotional problems?" It would be interesting to explore veteran use of AT by a disaggregated form of type of disability (e.g. physical impairment, visual impairment, and auditory impairment). Similarly, it would be interesting to also disaggregate the type of assistive device. The HAAT model explains how the human, the activity, and the assistive device can interact differently. As cited earlier, research has shown that different races use different assistive devices at different rates. Furthermore, in this study, differences were observed between the Whites/Blacks in the general population and White/Black veterans (e.g. the human from the HAAT model); similarly, there can possibly be differences between the types of devices and the types of disabilities. Future research should consider not delimiting the wealth of knowledge that can be obtained about veterans and the use of AT by disaggregating disability types and the various types of health equipment used.

\section{Conclusions}

Exploration of the relationship between AT use and veterans revealed racial differences between White and Black veterans based on socioeconomic determinants, access to health care, general health, and disability status. Different predictors and magnitudes of AT use were observed for White veterans opposed to Black veterans. Racial differences can partially be explained by components of the HAAT model such as the type of activity (e.g. employment) that the human is engaging in and the context (e.g. education and health coverage). The differences observed have many policy implications that can narrow the gap in utilization of AT between Black and White veterans. There is a need for the Veterans Administration to expand AT services to facilities primarily used by veterans. The expansion will increase awareness about the different types of devices, and inform veterans about resources including accessibility and affordability. Because our findings revealed that the presence of health coverage substantially increased the odds of using AT among veterans, service expansion should be combined with providing health benefits to narrow the gap in utilization of AT. There is also a policy needed to focus on increased education and cultural competency for health care providers. Being knowledgeable about the racial differences in utilization as well as being aware about the perceived barriers in use can lead to health care providers making informed decisions when encountering veteran patients. Lastly, we urge Congress to continue to recognize the benefits of AT in legislation. The most recent reauthorization of the Tech Act occurred in 2004. However, with the recogni- 
tion of racial differences in the utilization of AT in recent studies, there is a need for the establishment of executive statutes and regulations for groups who continually do not fully take advantage of available resources. Implementing additional policies specifically for the use of AT in the minority population may aid in increasing overall use among minority veterans.

\section{Conflict of interest}

The authors have no conflict of interest and are responsible for the content of this article.

\section{References}

Agee, E., Freedman, V., \& Cirbnabm, J. (2005). Reconsidering substation in long-term care. When does assistive technology take the place of personal care? Journal of Gerontology, 60, 272-280.

Alston, R., Lewis, A., \& Loggins, S. (2014). Assistive technology and veterans with severe disabilities: Examining the relationships among race, personal factors, medical support, income support, and use. Medical Care, 52, S17-S24.

Bailey, R. (1996). Human Performance Engineering (3rd ed.). Englewood Cliffs, NJ: Prentice-Hall.

Centers for Disease Control and Prevention (2011). BRFSS 2012 Survey Data and Documentation. Retrieved from http://www.cdc.gov/brfss. Updated August 23, 2013.

Cook, A., \& Hussey, S. (2002). Assistive technologies principles and practice (2nd ed.). St. Louis: Mosby.

Cornman, J. C., \& Freedman, V. A. (2008). Racial and ethnic disparities in mobility device use in late life. Journal of Gerontology: Social Sciences, 63B, S34-S41.
Erickson, W., Lee, C., \& von Schrader, S. (2014). 2012 Disability Status Report: United States. Ithaca, NY: Cornell University Employment and Disability Institute (EDI).

Kaye, H., Yeager, P., \& Reed, M. (2008). Disparities in usage of assistive technology among people with disabilities. Assistive Technology, 20, 194-203.

Lenker, J., \& Paquet, V. (2003). A review of conceptual models for assistive technology outcomes research and practice. Assistive Technology: The Official Journal of RESNA, 15(1), 1-15.

Lewis, A. (2009). Disability disparities: A beginning model. Disability Rehabilitation, 31, 1136-1143.

Lewis, A., Cooper, R., Seelman, K., et al. (2012). Assistive technology in rehabilitation: Improving impact through policy. Rehabilitation Research Policy Education, 2(26), 19-32.

Loggins, S., Alston, R., Lewis, A. (2014). Utilization of assistive technology by persons with physical disabilities: An examination of predictive factors by race. Disability Rehabilitation: Assistive Technology, 9(6), 487-492.

Murdoch, M., Hodges, J., Cowper, D., Fortier, L., \& van Ryn, M. (2003). Racial disparities in VA service connection for posttraumatic stress disorder disability. Medical Care, 41(4), 536-549.

National Consortium on Leadership and Disability for Youth (NCLD) (2007). Disability history timeline: Resource and discussion guide. Institute for Educational Leadership, Inc. ISBN 1-933493-20-8.

Public Law 100-407. The Technology-Related Assistance for Individuals with Disabilities Act of 1988.

Reed, K. (1992). History of Federal Legislation for persons with disabilities. The American Journal of Occupational Therapy, 46, 397-408.

Resnik, L., \& Allen, S. (2006). Racial and ethnic differences in use of assistive devices for mobility: Effect modification by age. Journal of Aging and Health, 18, 106-124.

Tomita, M. R., Mann, W. C., Fraas, L. F. \& Burns, L. L. (1997). Racial differences of frail elders in assistive technology. Assistive Technology, 9, 140-151. 